\title{
Magnetic anisotropies in ultrathin iron films grown on the surface-reconstructed GaAs substrate
}

\author{
B. Aktaş \\ Gebze Institute of Technology, 41400 Gebze-Kocaeli, Turkey \\ B. Heinrich, ${ }^{\text {a) }}$ G. Woltersdorf, and R. Urban \\ Simon Fraser University, Burnaby, British Columbia V5A 1S6, Canada \\ L. R. Tagirov \\ Gebze Institute of Technology, 41400 Gebze-Kocaeli, Turkey and Kazan State University, \\ 420008 Kazan, Russian Federation \\ F. Yıldız, K. Özdoğan, M. Özdemir, O. Yalçin, and B. Z. Rameev \\ Gebze Institute of Technology, 41400 Gebze-Kocaeli, Turkey
}

(Received 5 March 2007; accepted 10 May 2007; published online 11 July 2007)

\begin{abstract}
Magnetic anisotropies of epitaxial ultrathin iron films grown on the surface-reconstructed $\mathrm{GaAs}$ substrate were studied. Ferromagnetic resonance technique was exploited to determine magnetic parameters of the films in the temperature range of 4-300 K. Extraordinary angular dependence of the FMR spectra was explained by the presence of fourfold and twofold in-plane anisotropies. A strong in-plane uniaxial anisotropy with magnetic hard axis along the [1 $\overline{1} 0]$ crystallographic direction is present at the $\mathrm{GaAs} / \mathrm{Fe}(001)$ interface while a weak in-plane uniaxial anisotropy for the $\mathrm{Fe}$ grown on $\mathrm{Au}$ has its easy axis oriented along [1ㅣㅣ․ A linear dependence of the magnetic anisotropies as a function of temperature suggests that the strength of the in-plane uniaxial anisotropy is affected by the magnetoelastic anisotropies and differential thermal expansion of contacting materials. (C) 2007 American Institute of Physics. [DOI: 10.1063/1.2749469]
\end{abstract}

\section{INTRODUCTION}

The interest in ultrathin magnetic multilayers has been steadily increasing since they are building blocks in spintronics applications such as data storage devices and magnetic random access memories. The magnetic anisotropies of thin films are of crucial importance in understanding the physics of magnetic nanostructures. Ferromagnetic resonance (FMR) is a very accurate and straightforward technique, allowing one to determine magnetic anisotropy fields of ultrathin magnetic films. ${ }^{1,2}$ In this paper, we study the magnetic anisotropies in single GaAs/15Fe/20Au(001), GaAs/15Fe/20Cr(001), $\mathrm{GaAs} / 16 \mathrm{Fe} / 9 \mathrm{Pd} / 20 \mathrm{Au}(001)$, and double $\mathrm{GaAs} / 15 \mathrm{Fe} / \mathrm{Au} /$ $40 \mathrm{Fe} / 20 \mathrm{Au}(001)$ iron layer structures grown on the surfacereconstructed $\mathrm{GaAs}(001)$ single-crystalline substrate wafers. The integers represent the number of atomic layers. It will be shown that the interface-induced anisotropies can be used to tailor the overall magnetic properties of ultrathin film structures. In our FMR experiments, we observed unconventional triple-mode FMR spectra allowing one to discriminate between various in-plane magnetic anisotropies. Computer fitting of the angular and frequency dependent FMR spectra in the temperature range of $4-300 \mathrm{~K}$ allowed us to determine the cubic, uniaxial, and perpendicular components of the magnetic anisotropies and establish directions of the easy and hard axes in the individual layer(s). The magnetic anisotropies are discussed in terms of the interface and bulk anisotropies including magnetoelastic energy arising due to

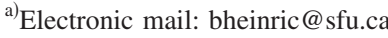

the lattice mismatch and differential thermal expansion of the metallic materials employed in these structures.

\section{SAMPLE PREPARATION}

Single $\quad 20 \mathrm{Au} / 15 / \mathrm{Fe} / \mathrm{GaAs}(001), \quad 30 \mathrm{Au} / 15 \mathrm{Fe} /$ $\mathrm{GaAs}(001), \quad 20 \mathrm{Cr} / 15 \mathrm{Fe} / \mathrm{GaAs}(001), \quad 20 \mathrm{Au} / 9 \mathrm{Pd} / 16 \mathrm{Fe} /$ $\mathrm{GaAs}(001)$, and double $20 \mathrm{Au} / 40 \mathrm{Fe} / 40 \mathrm{Au} / 15 \mathrm{Fe} / \mathrm{GaAs}(001)$ iron layer ultrathin film structures were prepared by molecular beam epitaxy $(\mathrm{MBE})$ on $(4 \times 6)$ reconstructed $\mathrm{GaAs}(001)$ substrates. The integers represent the number of atomic layers. A brief description of the sample preparation procedure is as follows. The $\mathrm{GaAs}(001)$ single-crystalline wafers were sputtered under grazing incidence using $600 \mathrm{eV}$ argon-ion gun to remove native oxides and carbon contaminations. Substrates were rotated around their normal during sputtering. After sputtering the GaAs substrates were annealed at approximately $580-600{ }^{\circ} \mathrm{C}$ and monitored by means of reflection high energy electron diffraction (RHEED) until a well-ordered $(4 \times 6)$ reconstruction appeared. ${ }^{3}$ The $(4 \times 6)$ reconstruction consists of $(1 \times 6)$ and $(4 \times 2)$ domains: the $(1 \times 6)$ domain is As-rich, while the $(4 \times 2)$ domain is $\mathrm{Ga}$ rich.

The Fe films were deposited directly on the GaAs(001) substrate at room temperature from a resistively heated piece of $\mathrm{Fe}$ at the base pressure of $1 \times 10^{-10}$ Torr. The film thickness was monitored by a quartz crystal microbalance and by means of RHEED intensity oscillations. The deposition rate was adjusted at about $1 \mathrm{ML}$ (monolayer)/min. The gold layer was evaporated at room temperature at the deposition rate of about $1 \mathrm{ML} / \mathrm{min}$. The RHEED oscillations were visible for 


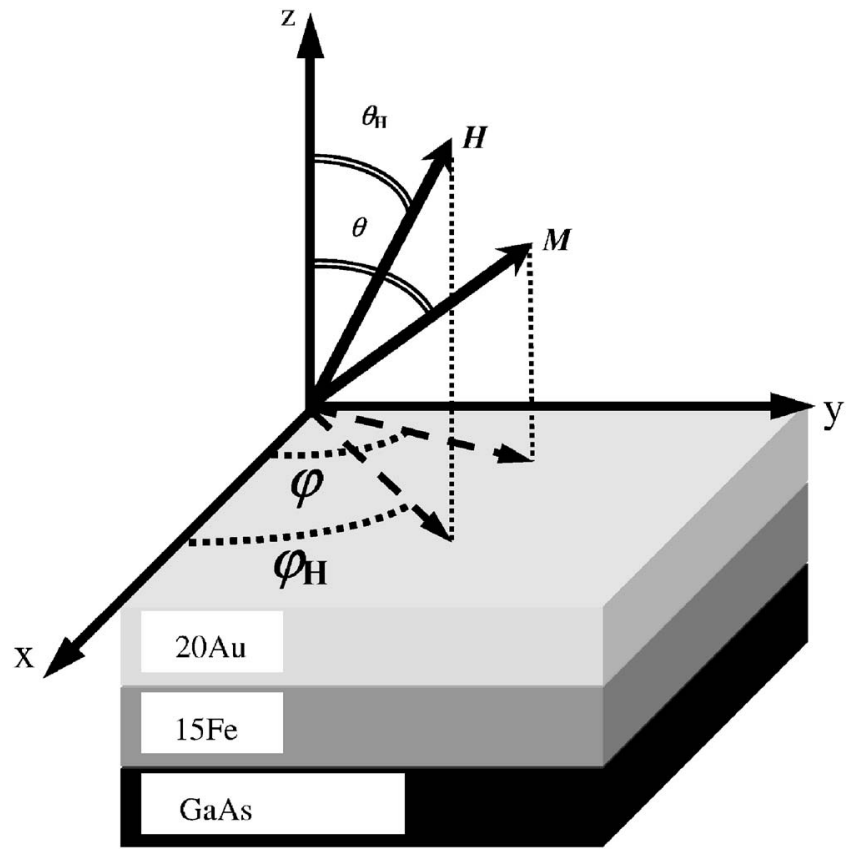

FIG. 1. The sketch of the samples studied in the paper.

up to 30 atomic layers. Films under study were covered by a 20-ML-thick $\mathrm{Au}(001)$ or $\mathrm{Cr}$ cap layer for protection in ambient conditions. More details of the sample preparation are given in Ref. 3 .

\section{MODEL AND BASIC FORMULAS FOR FERROMAGNETIC RESONANCE}

The FMR data are analyzed using the free energy expansion similar to that employed in Ref. 4,

$$
\begin{aligned}
E_{T}= & -\mathbf{M} \cdot \mathbf{H}+\left(2 \pi M_{0}^{2}-K_{p}\right) \alpha_{3}^{2}+K_{1}\left(\alpha_{1}^{2} \alpha_{2}^{2}+\alpha_{2}^{2} \alpha_{3}^{2}\right. \\
& \left.+\alpha_{3}^{2} \alpha_{1}^{2}\right)+K_{u} \cos ^{2}\left(\varphi-\varphi_{[1 \overline{1} 0]}\right) .
\end{aligned}
$$

The first term is the Zeeman energy in the external dc magnetic field, the second term is the demagnetization energy term including the effective perpendicular uniaxial anisotropy, the third term is the cubic anisotropy energy, and the last term is the in-plane uniaxial anisotropy energy with the symmetry axis along the [1 10$]$ crystallographic direction. ${ }^{5}$ $\alpha_{i}$ 's represent the directional cosines ${ }^{6}$ of the magnetization vector $\mathbf{M}$ with respect to the crystallographic axes ([100], [010] and [001]) of the $\mathrm{Fe}(001)$ film [or GaAs(001) substrate], and $M_{0}$ is the saturation magnetization. The relative orientation of the reference axes, sample sketch, and various vectors relevant in the problem are given in Fig. 1. The FMR condition is obtained by using a well known equation, ${ }^{7}$

$$
\begin{aligned}
\left(\frac{\omega_{0}}{\gamma}\right)^{2}= & \left(\frac{1}{M_{0}} \frac{\partial^{2} E_{T}}{\partial \theta^{2}}\right)\left(\frac{1}{M_{0} \sin ^{2} \theta} \frac{\partial^{2} E_{T}}{\partial \varphi^{2}}\right) \\
& -\left(\frac{1}{M_{0} \sin \theta} \frac{\partial^{2} E_{T}}{\partial \varphi \partial \theta}\right)^{2},
\end{aligned}
$$

where $\omega_{0}=2 \pi \nu$ is the circular frequency (determined by the operating frequency $\nu$ of the ESR spectrometer), $\gamma$ is the gyromagnetic ratio, and $\theta$ and $\varphi$ are the polar and azimuthal angles of the magnetization vector $\mathbf{M}$ with respect to the reference axes. The absorbed magnetic energy is caused by the Gilbert damping and is proportional to the out-of-phase rf susceptibility. ${ }^{1}$ Standing spin-wave excitations in our films were not considered because the film thickness is too small ( $\sim 20-80 \AA)$. The strength of magnetic anisotropies is obtained by computer fitting of the experimental data using Eq. (2).

In the in-plane FMR studies, the polar $\theta$ and $\theta_{H}$ angles were fixed at $\theta, \theta_{H}=\pi / 2$. The azimuthal angle of magnetization $\varphi$ was obtained from the static equilibrium condition for the given angle $\varphi_{H}$ of the external magnetic field. The angle $\varphi_{H}$ was varied from zero to $\pi$. Then, the set of equations for the in-plane geometry reads

$$
\begin{aligned}
& H \sin \left(\varphi-\varphi_{H}\right)+\frac{1}{2} H_{1} \sin 4 \varphi-H_{u} \sin 2\left(\varphi-\varphi_{[1 \overline{10}]}\right)=0 \\
&\left(\frac{\omega_{0}}{\gamma}\right)^{2}= {\left[H \cos \left(\varphi-\varphi_{H}\right)+4 \pi M_{\mathrm{eff}}+\frac{1}{2} H_{1}(3+\cos 4 \varphi)\right.} \\
&\left.-2 H_{u} \cos ^{2}\left(\varphi-\varphi_{[1 \overline{10}]}\right)\right]\left[H \cos \left(\varphi-\varphi_{H}\right)\right. \\
&\left.+2 H_{1} \cos 4 \varphi-2 H_{u} \cos 2\left(\varphi-\varphi_{[1 \overline{1} 0]}\right)\right]
\end{aligned}
$$

The effective magnetization $M_{\text {eff }}$ includes contribution from the perpendicular anisotropy: $2 \pi M_{\text {eff }}=2 \pi M_{0}-K_{p} / M_{0}$. The anisotropy fields are defined as follows: $H_{1}=K_{1} / M_{0}, H_{u}$ $=K_{u} / M_{0}$. The angle $\varphi_{[1 \overline{10}]}=\pi / 4$ is the angle between the easy direction of the cubic and hard axes of the uniaxial anisotropy.

For the out-of-plane FMR, the azimuthal angle $\varphi_{H}$ is fixed either at $\varphi_{H}=3 \pi / 4$ [easy axis, i.e., the dc magnetic field was rotated in the $(1 \overline{10})$ plane] or $\varphi_{H}=\pi / 4$ [hard axis, i.e., the dc magnetic field was rotated in the (110) plane], while the polar angle $\theta_{H}$ was varied from zero to $\pi / 2$. The polar and azimuthal angles of the magnetization were obtained from the static equilibrium condition corresponding to the minimum free energy of the system. The set of equations for the out-of-plane measurements from the easy axis direction reads

$$
\begin{aligned}
H \sin (\theta- & \left.\theta_{H}\right)-2 \pi M_{\mathrm{eff}} \sin 2 \theta \\
+ & \frac{1}{4} H_{1} \sin 2 \theta(3 \cos 2 \theta+1)=0 \\
\left(\frac{\omega_{0}}{\gamma}\right)^{2}= & {\left[H \cos \left(\theta-\theta_{H}\right)-4 \pi M_{\mathrm{eff}} \cos 2 \theta\right.} \\
& \left.+\frac{1}{2} H_{1}(\cos 2 \theta+3 \cos 4 \theta)\right] \\
& \times\left[H \cos \left(\theta-\theta_{H}\right)-4 \pi M_{\mathrm{eff}} \cos ^{2} \theta\right. \\
& \left.+\frac{1}{4} H_{1}\left(8 \cos 2 \theta-3 \sin ^{2} 2 \theta\right)+2 H_{u}\right] .
\end{aligned}
$$




\section{EXPERIMENTAL RESULTS}

\section{A. General measurement procedure}

FMR measurements were carried out using a commercial Bruker EMX $X$-band ESR spectrometer equipped by an electromagnet which provides a dc magnetic field up to $22 \mathrm{kG}$ in the horizontal plane. The FMR measurements were carried out in the range of $9.5 \mathrm{GHz}$. A small amplitude modulation of the dc field is employed to increase the signal to noise ratio. The field-derivative absorption signal was recorded in the temperature range of 4-300 K. An Oxford Instruments continuous helium-gas flow cryostat was used for cooling. The temperature was controlled by a commercial LakeShore 340 temperature-control system. A goniometer was used to rotate the sample around the sample holder in the cryostat tube. The sample holder was perpendicular to the dc magnetic field and parallel to the microwave magnetic field. The samples were placed on the sample holder in two different configurations. For the in-plane angular studies, the film was attached horizontally at the bottom edge of the sample holder. During rotation, the normal to the film plane remained parallel to the microwave field, but the external dc magnetic pointed along different directions with respect to the sample axes. This geometry is not conventional and gives an admixture of the in- and out-of-phase rf susceptibility components. Symmetric FMR peaks were obtained in conventional geometry in which the dc and microwave magnetic fields are always in the film plane. Some FMR measurements were done in conventional geometry which have shown that the FMR fields obtained in unconventional geometry do not differ from those obtained in conventional geometry. For the out-of-plane FMR measurements, the samples were attached to a flat platform which was cut with the normal perpendicular to the sample holder. Upon rotation of the sample holder, the microwave component of the field remained always in the sample plane, whereas the dc field was rotated from the sample plane toward the film normal.

\section{B. FMR in the single ferromagnetic layer samples \\ 1. In-plane FMR measurements}

For sample $20 \mathrm{Au} / 15 \mathrm{Fe} / \mathrm{GaAs}(001)$, Fig. 2(a) illustrates the temperature dependence of the in-plane FMR spectra for the dc magnetic field $\mathbf{H} \|[110]$. A single and relatively narrow FMR signal was observed at very low magnetic fields in the entire temperature range. Starting from $300 \mathrm{~K}$, the resonance field steadily shifted from $\sim 320$ down to about $150 \mathrm{G}$ at $5 \mathrm{~K}$. The FMR linewidth increased with decreasing temperature.

Contrarily, the measurements along the [110] direction have shown that the in-plane FMR spectrum unexpectedly consists of three signals [labeled by $P_{1}, P_{2}$, and $P_{3}$, Fig. 2(b)]. As far as we know, it is a unique observation. Usually, a single resonance or two resonance peaks are expected from ultrathin-film (15 ML) ferromagnetic layers (see, for example, Refs. 8-11 and also in Ref. 2, Figs. 2 and 3). Spinwave modes in ultrathin films are not observable in this frequency range. The temperature evolution of the FMR spectrum along the $[1 \overline{1} 0]$ direction is shown in Fig. 2(b).
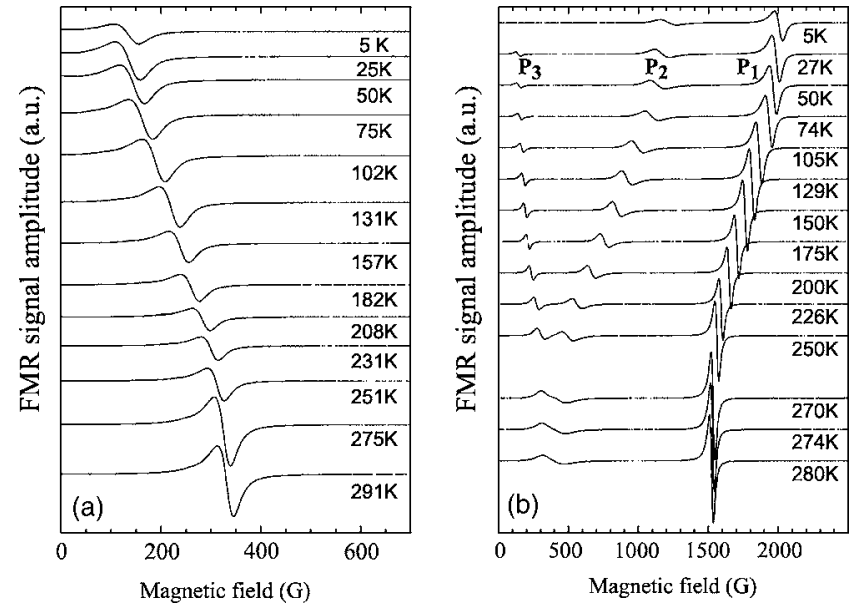

FIG. 2. Temperature dependence of the in-plane FMR spectra taken for $\mathbf{H} \|[110]$ (a) and $\mathbf{H} \|[1 \overline{1} 0]$ (b). Sample is $20 \mathrm{Au} / 15 \mathrm{Fe} / \mathrm{GaAs}(001)$.

These three peaks were present in the entire temperature range. The high-field signal has largest intensity at all temperatures. At room temperature (RT), the two low-field peaks overlapped and merged into the single, somewhat distorted FMR line. With decreasing temperature, the low-field signal separated into two signals, see Fig. 2(b). The high-field signal shifted gradually to higher fields upon lowering the temperature. At $T=4-5 \mathrm{~K}$, the splitting of the FMR peaks reached $\sim 1700 \mathrm{G}$. Notice that the high-field mode for $\mathbf{H} \|[1 \overline{1} 0]$ shifted in the opposite direction to the spectrum in the $\mathbf{H} \|[110]$ direction. This suggests that the easy magnetic axis is along the [110] crystallographic direction, and [110] is the hard magnetic axis.

The detailed study of magnetic anisotropies was carried out by rotating the dc magnetic field in the plane of the film. The angular dependence of FMR at RT is shown in Fig. 3. The number of absorption peaks was clearly varied with the in-plane angle of the dc field. The intensity of the FMR signals was also angular dependent. The overall angular periodicity is $180^{\circ}$. This implies that the sample has at least uniaxial in-plane symmetry. The unusual three-component FMR spectra require an additional anisotropy. It will be shown that the cubic anisotropy of $\mathrm{Fe}$ was needed to obtain the observed three-peak FMR spectra. The magnetic anisotropies obtained by FMR are sometimes frequency dependent. ${ }^{12}$ In order to check this point, the FMR measurements were also carried out in the frequency range of $9-36 \mathrm{GHz}$ at RT using our high-frequency extension modules. The right-hand-side inset in Fig. 3 shows the angular variation of the in-plane resonance field measured at $24 \mathrm{GHz}$. The left-hand-side inset shows the FMR field as a function of microwave frequency. A nearly parabolic dependence on the microwave frequency clearly indicates that the perpendicular anisotropy field $\left(4 \pi M_{\text {eff }}\right)$ is larger than the internal anisotropy fields in this frequency region. The anisotropy fields were found independent of the microwave frequency.

Computer fitting of the FMR data for the $20 \mathrm{Au} / 15 \mathrm{Fe} / \mathrm{GaAs}(001)$ allows one to determine the in-plane magnetic anisotropies. The results of this fitting are dis- 


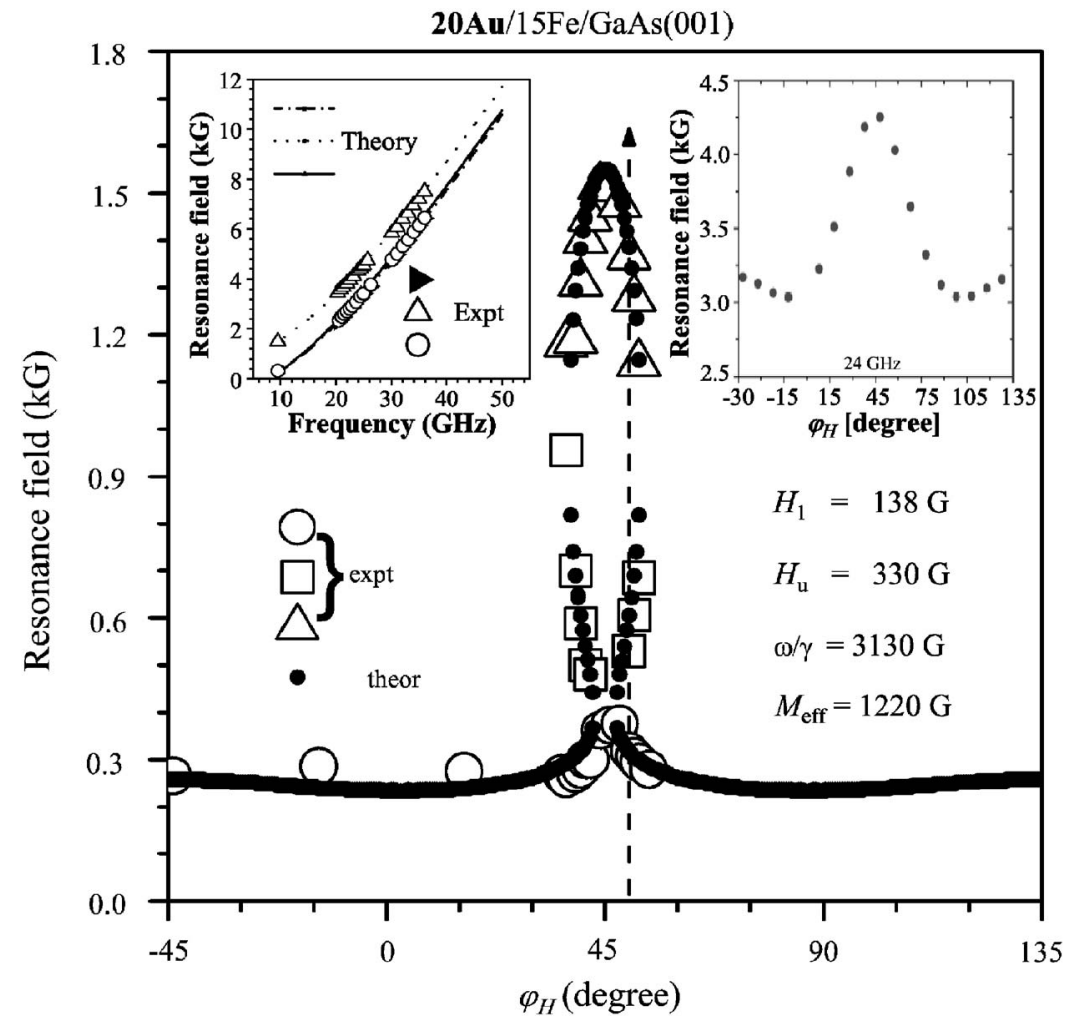

FIG. 3. In-plane angular dependence of FMR spectra for the $20 \mathrm{Au} / 15 \mathrm{Fe} / \mathrm{GaAs}(001)$ sample at room temperature and $\nu=9.497 \mathrm{GHz}$. Here and in Figs. 5 and 7,

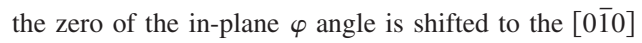
axis to bring the $[1 \overline{1} 0]$ hard-axis feature to the middle of the figure. Notice that the three FMR peaks are resolved for a narrow range of angles around the hard magnetic axis. Right-hand-side inset, the same angular dependence for $24 \mathrm{GHz}$, and left-hand-side inset, the frequency dependence of the resonance field measured for the easy (lower) and hard (upper) directions, respectively. played in Fig. 3. The calculated resonance fields $H$ $\equiv H_{\text {in }}^{\text {res }}$ ane are shown in solid circles and the measured FMR fields are represented by open symbols. The fitting parameters are given inside the figure. The in-plane angular dependence of the resonance field was fitted simultaneously with the out-of-plane FMR measurements (see next subsection).

The observed three FMR peaks in the vicinity of the magnetic hard axis are the consequence of competition between the cubic and uniaxial anisotropies with different directions of the easy axis. In zero dc field, the magnetization is along the axis corresponding to the minimum of energy. For $\left|K_{u}\right|>K_{1}$, the easy axis is along the [110] direction. By applying an external field along the hard axis [1 $1 \overline{10}]$, the magnetization started to rotate toward the closest [100] axis (the easy axis of the fourfold anisotropy) and consequently the uniaxial anisotropy decreased its energy, but the fourfold anisotropy and Zeeman energies got their contributions increased. The presence of three resonant peaks along the hard magnetic axis indicates that the competition between the uniaxial and fourfold anisotropies first results in an increase of the precessional frequency of FMR with increasing applied field, but the precessional frequency eventually reaches a maximum, $\omega_{\text {res }}$, and decreases when the magnetization is gradually rotated to the hard axis, see Fig. 4. When the magnetic moment is eventually aligned along the hard axis, then at that point the internal field is zero and consequently the precessional frequency is zero. The system becomes magnetically soft. The initial increase in the precessional frequency with increasing field is obvious from the right inset of Fig. 3. The resonant field corresponding to the magnetic moment oriented along [110] is higher than that required for the magnetic moment oriented along the [100] axis. This means that the precessional frequency along the easy axis

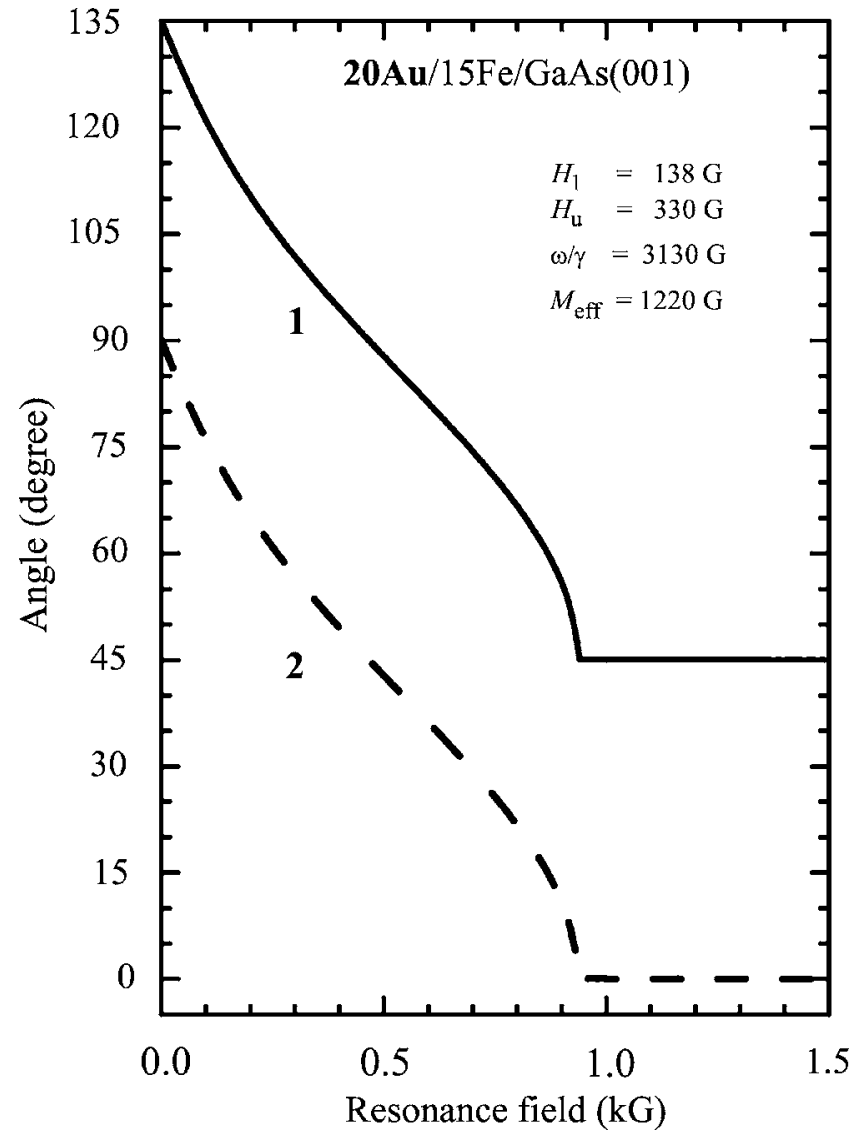

FIG. 4. Dependence of the magnetization angle on the applied magnetic field for the $20 \mathrm{Au} / 15 \mathrm{Fe} / \mathrm{GaAs}(001)$ sample at room temperature and $\nu$ $=9.497 \mathrm{GHz}$. The curve was drawn for the in-plane angle for the magnetic field $\varphi_{H}=45^{\circ}$. The curve labeled by " 1 " is the magnetization angle with respect to the easy axis [100] (corresponds to $\varphi=135^{\circ}$ ), while the curve labeled by " 2 " is the angle of magnetization with respect to the magnetic field applied at $\varphi_{H}=45^{\circ}$. 


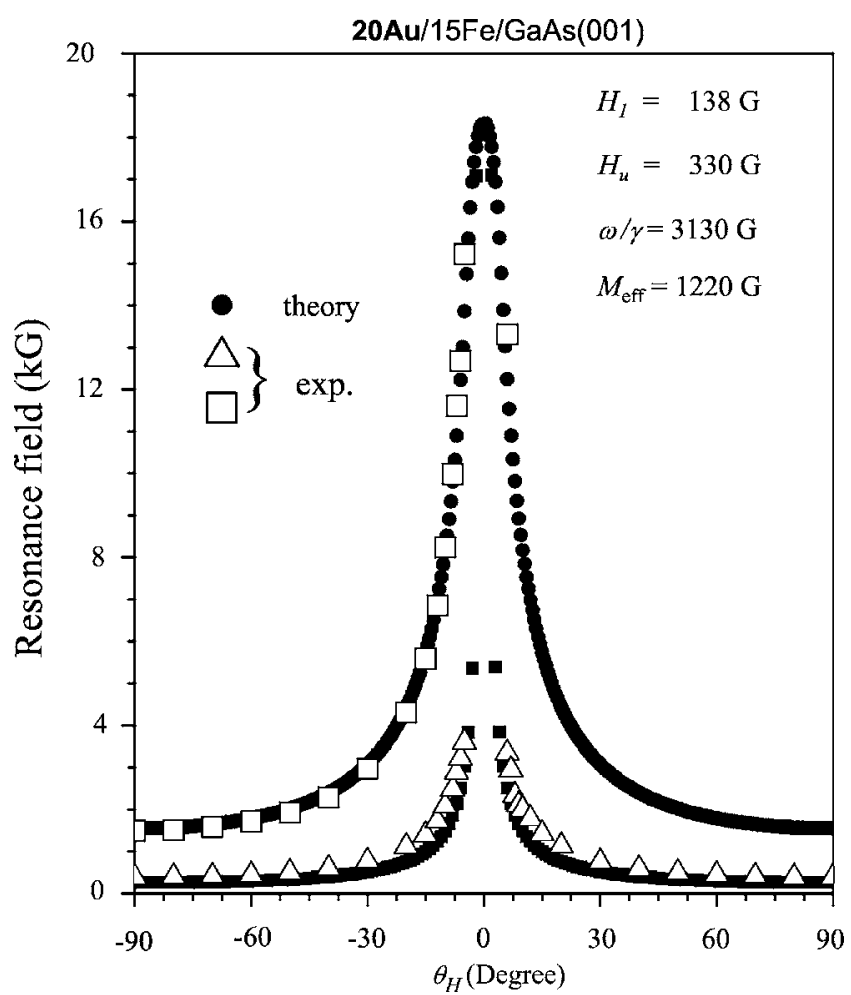

FIG. 5. Out-of-plane angular dependence of the resonance field for the $\mathrm{GaAs} / 15 \mathrm{Fe} / 20 \mathrm{Au}(001)$ sample. The dc field was applied in the (110) plane. The measurements were carried out at room temperature (RT).

[110] is lower than that along the [100] direction. In a narrow angle range, three resonant peaks can be observed, see Fig. 3 along the vertical dash line. The three peaks were observed when the angle between the magnetization and the [100] axis was in the range of $105^{\circ}-60^{\circ}$. The third peak, corresponding to the critical microwave resonant frequency, disappeared when the angle of the magnetization was very close to $90^{\circ}$ that corresponds to the cubic axis located between the easy [110] and hard [1 $\overline{1} 0]$ magnetic axes, see Fig. 4.

\section{Out-of-plane FMR measurements}

We have also made complementary out-of-plane FMR measurements when the dc magnetic field was rotated from the hard $[1 \overline{1} 0]$ axis in the film plane toward the normal direction to the film plane. The angular dependence of the FMR field as a function of the polar angle is shown in Fig. 5 by open squares and triangles. As expected, double-peak FMR spectra are observed for this geometry. The separation between the two modes steadily increases with approaching the film normal. The simultaneous fitting of the in- and outof-plane angular dependencies of the FMR spectra allow one to determine precisely the strength of crystalline anisotropies and $g$ factor.

The results of the fitting using Eqs. (4) for the out-ofplane FMR measurements of the $20 \mathrm{Au} / 15 \mathrm{Fe} / \mathrm{GaAs}(001)$ sample are plotted in Fig. 5 in solid circles. The fitting parameters are given in the figure. The out-of-plane FMR allows one to determine the $g$ factor. The calculations were done using $g=2.09$ and led to a fairly good agreement with the measurements at all angles in the in-plane and out-of-
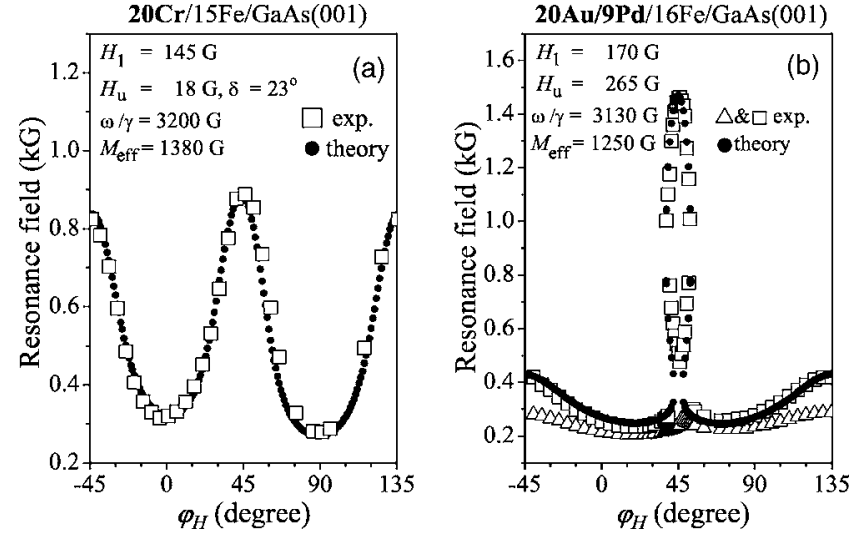

FIG. 6. Influence of the cap layer. The in-plane angular dependence of the resonance field: (a) chromium cap layer $(\nu=9.487 \mathrm{GHz})$ and (b) composite $\mathrm{Pd} / \mathrm{Au}$ cap layer $(\nu=9.510 \mathrm{GHz})$. The angle $\delta$ in the inset (a) is measured between the residual uniaxial anisotropy hard axis and the [100] axis. Measurements were carried out at RT.

plane geometries, and at all temperatures and frequencies. The temperature dependence of the magnetic anisotropies for the $20 \mathrm{Au} / 15 \mathrm{Fe} / \mathrm{GaAs}(100)$ and $30 \mathrm{Au} / 15 \mathrm{Fe} / \mathrm{GaAs}(001)$ samples is shown in Fig. 9(a).

\section{Influence of the cap layer material}

For the samples $20 \mathrm{Cr} / 15 \mathrm{Fe} / \mathrm{GaAs}(001)$ and $20 \mathrm{Au} / 9 \mathrm{Pd} / 16 \mathrm{Fe} / \mathrm{GaAs}(001)$, the in-plane geometry measurements have shown a drastic decrease of the uniaxial component in the in-plane anisotropy, see Fig. 6(a). A near absence of the in-plane uniaxial anisotropy in these measurements indicates that the $\mathrm{Cr}$ cap layer results in an almost complete canceling of the uniaxial anisotropy induced by the GaAs substrate. At the same time the principal axis of the residual uniaxial anisotropy is rotated about $23^{\circ}$ away from the original $[1 \overline{1} 0]$ direction corresponding to the $\mathrm{GaAs} / 15 \mathrm{Fe} / \mathrm{Au}(001)$ sample. This indicates that the presence of $\mathrm{Cr}$ overlayer resulted in two weak uniaxial in-plane anisotropies oriented along the [100] and [110] crystallographic directions. Another sample with a composite cap layer, $20 \mathrm{Au} / 9 \mathrm{Pd} / 16 \mathrm{Fe} / \mathrm{GaAs}(001)$, revealed only a minor influence of the palladium interlayer on the magnetic anisotropy of the iron film [see Fig. 6(b) and compare with Fig. 3]. However, the Fe film in $30 \mathrm{Au} / 15 \mathrm{Fe} / \mathrm{GaAs}(001)$ had a noticeably lower in-plane uniaxial anisotropy field than that in $20 \mathrm{Au} / 15 \mathrm{Fe} / \mathrm{GaAs}(001)$, showing again that the in-plane uniaxial anisotropy is dependent on the thickness of capping layer and is a complex property of the entire structure.

\section{FMR in the double-layer samples}

After measuring FMR in the single iron layers, we studied the spin-valve type, double-layer structures. Figure 7 shows temperature evolution of the in-plane FMR spectra for the $20 \mathrm{Au} / 40 \mathrm{Fe} / 40 \mathrm{Au} / 15 \mathrm{Fe} / \mathrm{GaAs}(001)$ sample. The data from the single-layer sample, $20 \mathrm{Au} / 15 \mathrm{Fe} / \mathrm{GaAs}(001)$, were used as a reference to identify the origin of the individual FMR peaks. The spectra in Fig. 7(a) have been recorded along the easy [110] axis for the first, 15-ML-thick iron layer, and the spectra in Fig. 7(b) have been recorded along 

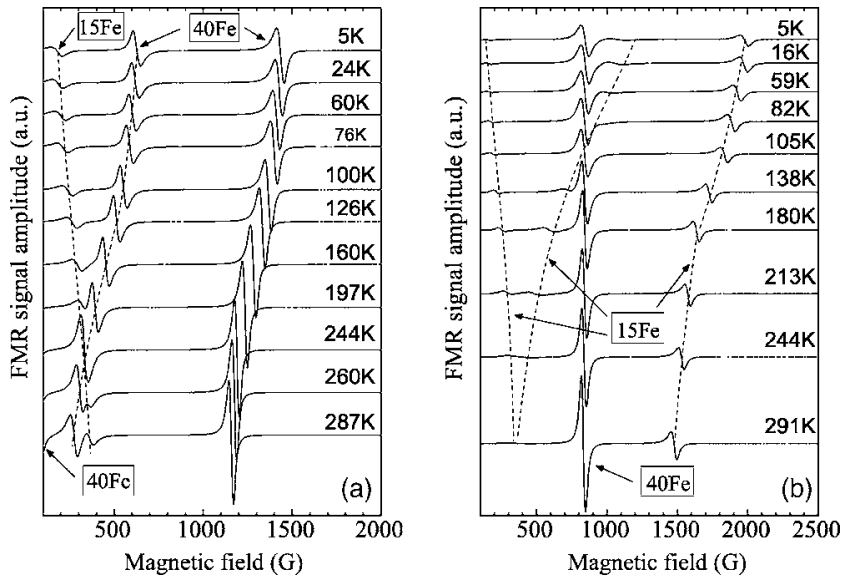

FIG. 7. The in-plane FMR spectra of the double-layer sample, $20 \mathrm{Au} / 40 \mathrm{Fe} / 40 \mathrm{Au} / 15 \mathrm{Fe} / \mathrm{GaAs}(001)$, for two orientations of magnetic field with respect to the crystallographic axes: (a) dc field is parallel to the easy axis of the first, 15-ML-thick iron layer; (b) dc field is parallel to the hard axis of the first layer. The dash lines are guides for the reader's eye. The measurements were carried out at $\nu=9.51 \mathrm{GHz}$.

the hard axis $[1 \overline{1} 0]$ of the first layer. The reference spectra from the $20 \mathrm{Au} / 15 \mathrm{Fe} / \mathrm{GaAs}(001)$ sample can be found in Figs. 2(a) and 2(b), respectively.

In the measured temperature range from 5 to $291 \mathrm{~K}$, three FMR absorption peaks were present for the spectra recorded at the [110] direction [Fig. 7(a)]. As we do not expect any marked interlayer exchange coupling or magnetostatic interaction through the 40-ML-thick gold layer, the contribution of the first, 15-ML-thick iron layer to the multicomponent FMR spectra can be easily identified [see labeling in Figure 7(a)] by comparison with the measurements on the single-layer sample, Fig. 2(a). A double peak spectra (at higher magnetic fields) from the 40-ML-thick layer allow one to conclude that the [110] direction is a hard magnetic axis for this layer. A partly developed third peak at very small fields is observed only close to room temperature. One can see only its tail, and that was in agreement with the calculated FMR peaks, see Fig. 8.

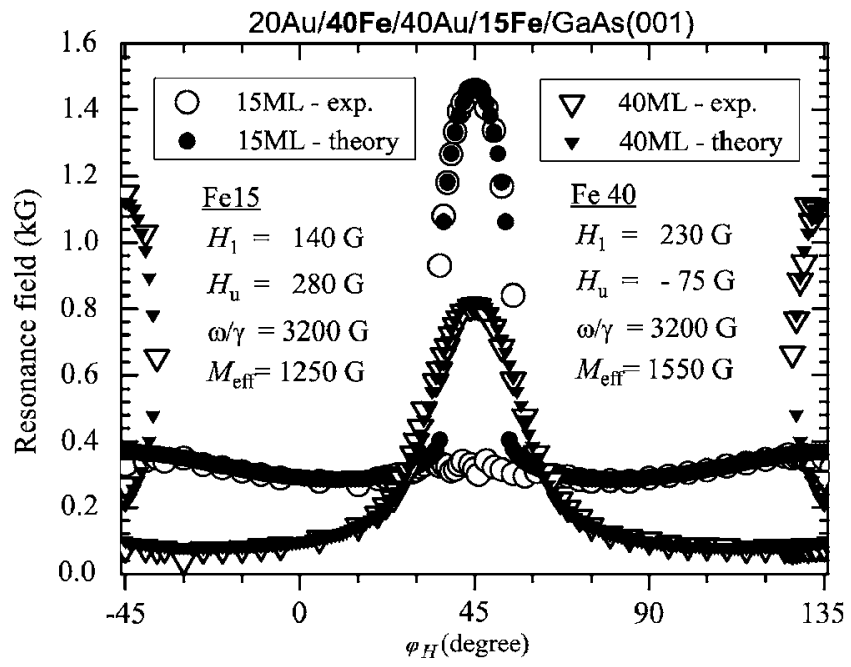

FIG. 8. The angular dependence of the in-plane resonance field for the double-layer sample at room temperature and $\nu=9.51 \mathrm{GHz}$ : open symbols, experimental data, and solid symbols, results of the fitting.
The fitting of the full angular dependence of FMR of the second $40 \mathrm{ML}$ layer revealed that the hard axis of the uniaxial anisotropy term in the second, 40-ML-thick iron layer, is switched $90^{\circ}$ with respect to the hard axis of that in the first, 15-ML-thick iron layer. The fitting parameters given in Fig. 8 have shown that the in-plane uniaxial anisotropy of the 40-ML-thick layer is drastically reduced and has the opposite sign compared with the first, 15-ML-thick iron layer.

The FMR spectra recorded along the $[1 \overline{1} 0]$ direction, see Fig. 7(b), show a four-peak structure in the main domain of temperatures. Three of them can be identified as a hard-axis spectra of the first, 15-ML-thick iron layer [see labeling in Fig. 7(b) and compare with Fig. 2(b)]. As expected, the single-peak FMR spectrum of the 40-ML-thick layer clearly indicates that $[1 \overline{1} 0]$ is the magnetic easy axis.

\section{Temperature dependence of the anisotropy fields and discussion of results}

The temperature dependence of the magnetic anisotropies are shown in Fig. 9. The effective magnetization $M_{\text {eff }}$ includes perpendicular anisotropy [see Eq. (1)], and therefore $M_{\text {eff }}$ is reduced compared with the bulk magnetization [ $\sim 1.71 \mathrm{kG}$ (Ref. 13)] by $\sim 400-500 \mathrm{G}$ at RT for the single iron layer samples. The effective magnetization increased with decreasing temperature. The Curie temperature of bulk iron is about $980^{\circ} \mathrm{C}$. Assuming that the 15 -ML-thick Fe film has its Curie point close to $980{ }^{\circ} \mathrm{C}$, the saturation magnetization would have been increased only by $\sim 64 \mathrm{G}$ in the temperature range from 300 to $5 \mathrm{~K}^{13}$ Therefore, the observed decrease in $M_{\text {eff }}$ by $\sim 300 \mathrm{G}$ would require that the temperature dependence of $M_{\text {eff }}$ had to be caused mostly by the decreasing value of the perpendicular uniaxial field with decreasing temperature. The uniaxial perpendicular anisotropy at RT is inversely proportional to the film thickness and therefore it is reasonable to assume that it originates from the broken symmetry at the $\mathrm{Fe} / \mathrm{GaAs}(001)$ and $\mathrm{Au} / \mathrm{Fe}(001)$ interfaces, see Ref. 14. The magnetic anisotropies in Fe usually increase with decreasing temperature. However, by cooling the strain in the film changed by differential thermal expan-

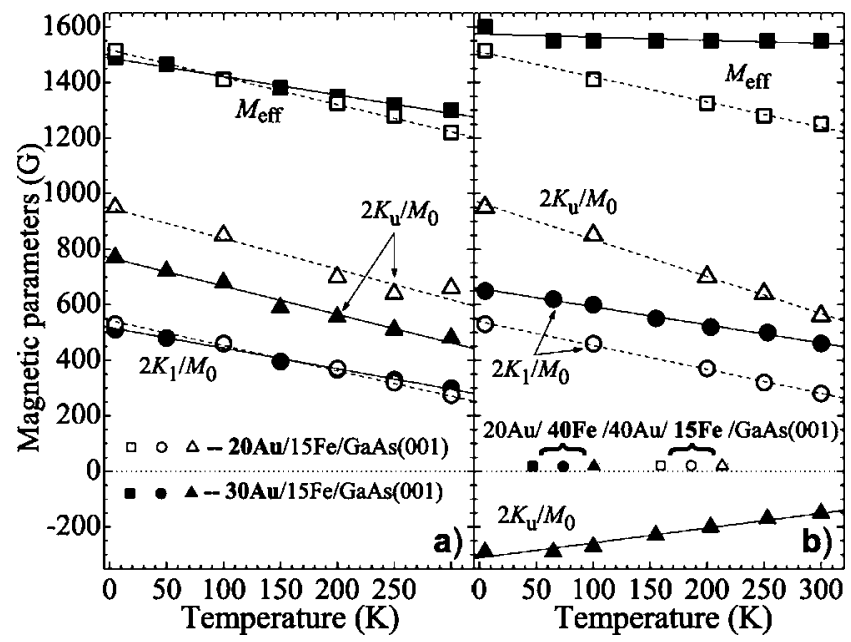

FIG. 9. Temperature dependence of the magnetic parameters: (a) for the single-layer sample and (b) for the double-layer sample. 
TABLE I. Thermal expansion and lattice parameters.

\begin{tabular}{lccclll}
\hline \hline & Thermal expan. & $\left(10^{-6} \mathrm{~K}^{-1}\right)$ & & \\
\cline { 2 - 5 } Material (structure) & $298 \mathrm{~K}$ & $523 \mathrm{~K}$ & $1273 \mathrm{~K}$ & $\begin{array}{l}\text { Lattice parameter } \\
a=b=c=(\AA)\end{array}$ & Effect on iron layer \\
\hline Iron (bcc-Fe) & 11.8 & 15.0 & 24.0 & 2.8665 & \\
GaAs(ZnS structure) & 5.73 & & & $5.654 / 2=2.827$ & Compressive strain \\
Gold (fcc-Au) & 14.2 & 14.6 & 16.7 & $4.078 / \sqrt{2}=2.892$ & Tensile strain \\
Chromium (bcc-Cr) & 6.2 & & & 2.91 & Large tensile strain \\
Palladium (fcc-Pd) & 11.8 & 12.2 & 13.9 & $3.891 / \sqrt{2}=2.759$ & Compressive strain \\
\hline \hline
\end{tabular}

sion, see Table I, can result in interface magnetoelastic contributions which can be responsible for the observed decrease of the uniaxial perpendicular anisotropy.

The values of the in-plane uniaxial and cubic anisotropies increase with decreasing temperature. The sign of the cubic anisotropy parameter is positive, making the principal crystalline directions $\langle 100\rangle$ easy magnetic axes. For the uniaxial anisotropy $K_{u}>K_{1}$, the in-plane magnetic hard axis is along the [110] crystallographic direction. The in-plane fourfold uniaxial anisotropy does not show any surprises as a function of the film thickness. It decreases with decreasing film thickness due to the presence of the interface fourfold anisotropy which has an opposite sign to that of the bulk cubic anisotropy.

The most surprising behavior is found for the in-plane uniaxial anisotropy. It is interesting to point out complexities related to the in-plane uniaxial anisotropy in $\operatorname{GaAs}(001)$ structures. ${ }^{15}$ The origin of the large in-plane interface uniaxial anisotropy in $\mathrm{GaAs} / \mathrm{Fe}(001)$ has so far not been clearly understood. The hard magnetic axis lies along the [110] crystallographic direction which is parallel to the dangling bonds of As terminated $(2 \times 6)$ and pseudo$(4 \times 6)$-reconstructed $\mathrm{GaAs}(001)$ substrates. ${ }^{3}$ However, Moosbühler et al. ${ }^{16}$ have shown that the strength and sign of the in-plane uniaxial surface anisotropy are not affected by a particular reconstruction of the GaAs template. A genuine Ga-rich $(4 \times 6)$ reconstruction results in almost the same uniaxial anisotropy as that observed in the $(2 \times 6)$ As-rich reconstruction. Therefore, it is hard to believe that the source of this anisotropy lies in the chemical bonding between the dangling bonds of As and Fe. This point of view is further supported by our results using $\mathrm{Cr}(001)$ overlayer. $\mathrm{Cr}(001)$ layer grown over a 15-ML-thick $\mathrm{Fe}(001)$ film can almost entirely remove the in-plane uniaxial anisotropy, see Fig. 6(a). The in-plane uniaxial anisotropy was also found to be dependent on the thickness of the capping Au layer in the 20,30Au/15Fe/GaAs(001) structures, see Fig. 9(a). These results imply again that the interface chemistry alone between the As and Fe interface atoms cannot be the source of the in-plane anisotropy. There is about $-1.5 \%$ misfit between lattice parameters of $\mathrm{Fe}, \mathrm{Au}$, and $\mathrm{GaAs}$ substrates. Fe films grown on $\mathrm{GaAs}(001)$ are under a compressive strain. Calculations by Mirbt et al. ${ }^{17}$ have suggested that an in-plane interface shear (of the order of $2 \%$ ) can be established at the $\mathrm{Fe} / \mathrm{GaAs}(001)$ structures. A significant in-plane lattice shear was observed by Xu et al. ${ }^{19}$ in $\mathrm{Fe} / \mathrm{InAs}(100)$ structures and Thomas et al. in relatively thick $\mathrm{Fe}$ films grown on
GaAs(001). ${ }^{18}$ The in-plane shear can lead to an in-plane uniaxial anisotropy due to the magnetoelastic parameter $B_{2}$ (Ref. 20) with the uniaxial magnetic axis oriented along one of the $\langle 110\rangle$ directions.

The in-plane uniaxial anisotropy in the $40 \mathrm{Fe}(001)$ film surrounded by the $\mathrm{Au}(001)$ layers $[20 \mathrm{Au} / 40 \mathrm{Fe} / 40 \mathrm{Au} / 15 \mathrm{Fe} / \mathrm{GaAs}(001)]$ changed the easy inplane uniaxial axis to [1힐. Au has a larger (001) square mesh than that of $\mathrm{Fe}(001)$ by $0.9 \%$. The $\mathrm{GaAs}(001)$ mesh is smaller by $1.4 \%$ than that of $\mathrm{Fe}(001)$, see Table I. The Fe film on $\mathrm{GaAs}(001)$ is under contraction while $\mathrm{Fe}$ on $\mathrm{Au}(001)$ is under tension. That can result in a reversal of the sign of interface shear in the $20 \mathrm{Au} / 40 \mathrm{Fe} / 40 \mathrm{Au}(001)$ structure compared to that at the GaAs/Fe(001) interface. This would imply that for $\mathrm{Fe} / \mathrm{Au}(001)$, the surface cell length of $\mathrm{Fe}$ along [110] would be larger than the surface cell length along [1]0]. Magnetoelastic coupling in $20 \mathrm{Au} / 40 \mathrm{Fe} / 40 \mathrm{Au}(001)$ can then lead to an interface uniaxial anisotropy with the easy axis along the $[1 \overline{1} 0]$ direction. The $\mathrm{Cr}(001)$ square mesh is $1.7 \%$ larger than that of $\mathrm{Fe}(001)$. This lattice mismatch is almost twice of that found in $\mathrm{Fe} / \mathrm{Au}(001)$. Therefore, one can argue that the shear at the $\mathrm{Fe} / \mathrm{Cr}(001)$ interface can be larger than that at the $\mathrm{Fe} / \mathrm{Au}(001)$. It can be argued that the shear at the $\mathrm{Fe} / \mathrm{Cr}(001)$ interface can result in a large enough in-plane uniaxial anisotropy compensating the in-plane uniaxial anisotropy from the $\mathrm{GaAs} / \mathrm{Fe}(001)$ interface in agreement with our measurements on the $20 \mathrm{Cr} / 15 \mathrm{Fe} / \mathrm{GaAs}(001)$ sample, see Fig. 6(a). However, the in-plane uniaxial anisotropy was not changed by a Pd layer, see Fig. 6(b). The Pd square mesh is $4.6 \%$ smaller than that of $\mathrm{Fe}$. Therefore, one can expect a larger in-plane uniaxial anisotropy in $20 \mathrm{Au} / 9 \mathrm{Pd} / 16 \mathrm{Fe} / \mathrm{GaAs}(001)$ compared to that measured in $\mathrm{Au} / \mathrm{Fe} / \mathrm{GaAs}(001)$. Only a marginal enhancement of 20\% was found, see Figs. 6(b) and 3. This can be caused by a large lattice mismatch between the $\mathrm{Fe}(001)$ and $\operatorname{Pd}(001)$ lattice meshes. Perhaps in this case the Pd square lattice mesh relaxes its strain right from the first atomic layer and consequently affects the interface shear only marginally.

It is interesting to note that all magnetic anisotropies were found linearly dependent on temperature within the experimental error, see Fig. 9. The almost linear temperature dependence of the perpendicular uniaxial anisotropy has been observed also in Ref. 21. This suggests that there could be common physical grounds behind this unified universal behavior. The strain between $\mathrm{Fe}(001)$ and $\mathrm{GaAs}(001)$ decreases by $\sim 40 \%$ from RT to $4 \mathrm{~K}$. This is an estimate based on using known thermal expansion coefficients for the bulk 
$\mathrm{Fe}, \mathrm{Au}$, and GaAs crystals, see Table I. The observed increase in the in-plane uniaxial anisotropy field by approximately $60 \%$ from RT to liquid He temperature, see Fig. 9, suggests that the difference in the surface cell length along [110] and [1 $\overline{1} 0]$ closely followed the relaxation of stress with decreasing temperature. One may attribute the temperature dependence of the magnetic anisotropies to the temperature dependence of the magnetoelastic parameters $B_{1}$ and $B_{2}$. This can be applicable to the in-plane and perpendicular uniaxial anisotropies. However, the same linear dependence on temperature was found also for the in-plane fourfold anisotropy, see Fig. 9 and yet there is no magnetoelastic term known for the fourfold magnetic anisotropy. ${ }^{15}$ Therefore, it is unlikely that the temperature dependence of the magnetoelastic energy on its own can be a possible explanation for the observed linear dependence of the fourfold magnetic anisotropy on temperature, as shown in Fig. 9.

\section{CONCLUSION}

We studied the magnetic anisotropies of epitaxial, crystalline ultrathin iron films grown on the surfacereconstructed $(4 \times 6) \mathrm{GaAs}(001)$ substrate. The ferromagnetic resonance technique has been explored extensively to determine magnetic parameters of the studied films in the temperature range from 4 to $300 \mathrm{~K}$. The triple-peak FMR spectra were observed, allowing an accurate extraction of magnetic anisotropies using computer simulations of the experimental data. The measured samples have shown strong perpendicular and in-plane uniaxial anisotropies in the $\mathrm{Au} / \mathrm{Fe} / \mathrm{GaAs}(001)$ films. The fourfold in-plane anisotropy decreases with the film thickness due to a presence of the interface fourfold contribution which has an opposite sign to that of the bulk cubic anisotropy. The most surprising behavior is found for the in-plane uniaxial anisotropy induced by reconstruction of the GaAs substrate surface. It is argued that the in-plane uniaxial anisotropy in $\mathrm{Au}, \mathrm{Cr}, \mathrm{Pd} / \mathrm{Fe} / \mathrm{GaAs}(001)$ and $\mathrm{Au} / \mathrm{Fe} / \mathrm{Au} / \mathrm{Fe} / \mathrm{GaAs}(001)$ structures is more likely affected by the interface shear strain. The experiment shows that the $\mathrm{Cr}(001)$ layer grown over a 15-ML-thick Fe(001) film can almost entirely remove the in-plane uniaxial anisotropy. The fitting of the angular dependence of FMR of the double magnetic layer sample, $20 \mathrm{Au} / 40 \mathrm{Fe} / 40 \mathrm{Au} / 15 \mathrm{Fe} /$ $\mathrm{GaAs}(001)$, revealed that the easy (hard) axis of the uniaxial anisotropy term in the second, 40-ML-thick iron layer is switched $90^{\circ}$ with respect to the easy (hard) axis of the 15-
ML-thick iron layer. It has been shown that the surface reconstruction of the GaAs substrate and various combination of materials in the multilayer structure can be used for tailoring of the magnetic anisotropies in spin-valve-like, double ferromagnetic layer structure.

\section{ACKNOWLEDGMENTS}

This work was supported in part by the Gebze Institute of Technology, Grant No 03-A12-1, and Russian Ministry of Education and Science. One of the authors (B.H.) thanks the Canadian National Science Engineering Research Council (NSERC) and Canadian Institute for Advanced Research (CIAR) for a generous and valuable scientific research support.

${ }^{1}$ B. Heinrich and J. F. Cochran, Adv. Phys. 42, 523 (1993).

${ }^{2}$ M. Farle, Rep. Prog. Phys. 61, 755 (1998).

${ }^{3}$ T. L. Monchesky, B. Heinrich, R. Urban, K. Myrtle, M. Klaua, and J. Kirshner, Phys. Rev. B 60, 10242 (1999).

${ }^{4}$ S. McPhail, C. M. Gürtler, F. Montaigne, Y. B. Xu, M. Tselepi, and J. A. C. Bland, Phys. Rev. B 67, 024409 (2003).

${ }^{5}$ M. Dumm, F. Bensch, R. Moosbühler, M. Zölfl, M. Brockmann, and G. Bayreuther, in Magnetic Storage Systems Beyond 2000, NATO Science Series II: Mathematics, Physics and Chemistry, edited by G. C. Hadjipanayis (Kluwer Academic, Dordrecht, 2001), Vol.41, pp. 555-558.

${ }^{6}$ A. G. Gurevich and G. A. Melkov, Magnetic Oscillations and Waves (CRC, New York, 1996), chap. 2.

${ }^{7}$ H. Suhl, Phys. Rev. 97, 555 (1955).

${ }^{8}$ J. J. Krebs, F. J. Rachford, P. Lubitz, and G. A. Prinz, J. Appl. Phys. 53, 8058 (1982)

${ }^{9}$ Yu. V. Goryunov, N. N. Garifyanov, G. G. Khaliullin, I. A. Garifullin, L. R. Tagirov, F. Schreiber, Th. Mühge, and H. Zabel, Phys. Rev. B 52, 13450 (1995).

${ }^{10}$ Th. Mühge et al., J. Appl. Phys. 81, 4755 (1997).

${ }^{11}$ T. Toliński, K. Lenz, J. Lindner, E. Kosubek, K. Baberschke, D. Spoddig, and R. Mechenstock, Solid State Commun. 128, 385 (2003).

${ }^{12}$ G. Woltersdorf and B. Heinrich, Phys. Rev. B 69, 184417 (2004).

${ }^{13}$ Numerical Data and Functional Relationships in Science and Technology, Landolt-Börnstein, New Series, Vol. III, Pt. 19A (Springer, Heidelberg, 1986)

${ }^{14}$ R. Urban, G. Woltersdorf, and B. Heinrich, Phys. Rev. Lett. 87, 217204 (2001).

${ }^{15}$ G. Wastlbauer and J. A. C. Bland, Adv. Phys. 54, 137 (2005).

${ }^{16}$ R. Moosbuehler, F. Bensch, M. Dumm, and G. Bayreuther, J. Appl. Phys. 91, 8757 (2002).

${ }^{17}$ S. Mirbt, B. Sanyal, C. Ischeden, and B. Johansson, Phys. Rev. B 67, $155421(2003)$

${ }^{18}$ O. Thomas, Q. Shen, P. Schieffer, N. Tournerie, and B. Lépine, Phys. Rev. Lett. 90, 017205 (2003).

${ }^{19}$ Y. B. Xu, D. J. Freeland, M. Tselepi, and J. A. Bland, Phys. Rev. B 62, 1167 (2002).

${ }^{20}$ D. Sander, Rep. Prog. Phys. 62, 809 (1999).

${ }^{21}$ Kh. Zakeri, Th. Kebe, J. Lindner, and M. Farle, J. Magn. Magn. Mater. 299, L1 (2005); Phys. Rev. B 73, 052405 (2006). 